www.nature.com/clinicalpractice/uro

\section{Technical preferences for hypospadias repair}

Numerous techniques exist for the surgical repair of hypospadias, with considerable controversy about which is the optimal method, yet little has been published regarding which techniques are used most often by practicing clinicians. Now Cook and colleagues in Canada have conducted a survey to determine which surgical techniques are preferred by an international cohort of pediatric urologists.

The investigators developed a questionnaire presenting five hypothetical hypospadias cases including distal, midshaft, and proximal defects associated with varying degrees of CHORDEE. Participants were asked to indicate which repair technique they would use in each scenario and what factors influenced this decision. Relevant demographic data was also requested. The questionnaire was sent to 121 pediatric urologists, of which 101 responded.

The majority of respondents preferred the tubularized incised urethral plate (TIP) technique for the repair of both distal and midshaft hypospadias, whereas a variety of techniques were favored for proximal defects and chordee correction. The most important factor influencing choice of technique was personal experience.

Statistical analysis using Spearman's rank correlation coefficient showed that choice of repair technique was not influenced by the number of hypospadias repairs performed monthly, the type of practice (academic versus private), or the number of years in practice.

The authors conclude that prospective, comparative trials are needed to determine the optimal surgical technique for hypospadias repair.

\section{Tamsin Osborne}

Original article Cook A et al. (2005) A multicenter evaluation of technical preferences for primary hypospadias repair. J Urol 174: 2354-2357

\section{Ciclosporin is successful in the treatment of interstitial cystitis}

Retrospective analysis has shown ciclosporin to be an effective and well-tolerated oral therapy for the treatment of interstitial cystitis, but prospective studies are lacking. Sairanen et al. conducted a prospective, multicenter, randomized, open-label Finnish trial comparing ciclosporin with pentosan polysulfate sodium (PPS), which is approved by the FDA for the treatment of interstitial cystitis.

In total, 64 (11 male) patients were randomized to receive either ciclosporin $1.5 \mathrm{mg} / \mathrm{kg}$ twice daily $(n=32)$ or PPS $100 \mathrm{mg}$ three times daily for 6 months. In the case of four patients who reported adverse events, the ciclosporin dose was halved. Ciclosporin was superior to PPS in all clinical outcome parameters when measured at 6 months. Micturition frequency in $24 \mathrm{~h}$ was significantly improved in the ciclosporin group $(-6.7 \pm 4.7$ times), compared with the PPS group $(-2.0 \pm 5.1$ times, $P<0.001)$. In addition, the proportion of responders increased to $75 \%$ over 6 months in the ciclosporin group, while it remained stable in the PPS group.

Further investigation is warranted, as more patients in the ciclosporin group experienced adverse effects, including increased blood pressure and serum creatinine levels. The authors conclude that, nonetheless, ciclosporin was more effective than PPS in the treatment of interstitial cystitis. They recommend ciclosporin for patients with severe interstitial cystitis, especially those who have not benefited from other therapies.

Katherine Sole

Original article Sairanen J et al. (2005) Cyclosporine A and pentosan polysulfate sodium for the treatment of interstitial cystitis: a randomized comparative study. J Urol 174: 2235-2238

\section{Phase II trial shows carboxyamidotriazole is ineffective in refractory $\mathrm{RCC}$}

Phase I trials have suggested that the antiangiogenic agent carboxyamidotriazole might stabilize the progression of advanced renal cell carcinoma (RCC) after first-line immunotherapy fails. Results of the Phase II Eastern Cooperative Oncology Group study show that this is not the case; the authors say that the apparent stabilization previously seen is the result of underestimation of the expected survival time for patients with advanced RCC in second-line clinical trials.

Carboxyamidotriazole $250 \mathrm{mg}$ daily was given to 53 patients with advanced, recurrent or metastatic RCC who had experienced disease progression after previous treatment with at least one immunotherapeutic agent.

\section{GLOSSARY}

CHORDEE

Ventral curvature

of the penis, as seen

in hypospadias due

to congenital shortness

of the ventral skin 